\title{
牡蚛壳为原料制备医用 $\mathrm{CaCO}_{3} / \mathrm{HA}$ 复合生物材料
}

\author{
刘子阳，耿振，李朝阳 \\ (天津大学 材料科学与工程学院, 天津 300350)
}

摘 要: 以牡蚛壳为原材料, 通过水热法制备了碳酸钲 $\left(\mathrm{CaCO}_{3}\right)$ 羟基磷灰石 $(\mathrm{HA})$ 复合材料, 拟达到降低 $\mathrm{HA}$ 生产成 本并改善其降解性能的目的。通过物相分析和 SEM、TEM 观察发现制得的 $\mathrm{CaCO}_{3} / \mathrm{HA}$ 复合材料呈现片层状，其微 观形貌呈现纳米颗粒状。实验通过控制钙、磷元素的投料比例制备了 $\mathrm{HA}$ 含量为 $20 \% 、 40 \% 、 60 \%$ 的三种 $\mathrm{CaCO}_{3} / \mathrm{HA}^{\circ}$ 复合材料 $(20 \% \mathrm{HA} 、 40 \% \mathrm{HA} 、 60 \% \mathrm{HA})$ ，通过 ICP 测试计算得出 HA 的实际含量为 $17.52 \% 、 34.30 \% 、 43.24 \%$ 。随着 $\mathrm{HA}$ 含量的增加, $\mathrm{CaCO}_{3} / \mathrm{HA}$ 复合材料的比表面积和热稳定性显著提升。体外降解实验结果表明, 三种不同 $\mathrm{HA}$ 含量 的复合材料在 PBS 模拟体液中 $14 \mathrm{~d}$ 的降解率分别为 $15.2 \%, 12.0 \%$ 和 $10.8 \%$, 降解率随 HA 比例的增高而降低。这 些结果表明: 水热法合成 $\mathrm{CaCO}_{3} / \mathrm{HA}$ 复合材料可通过钙、磷元素的投料比例来调控 $\mathrm{HA}$ 的转化率，进而调控 $\mathrm{CaCO}_{3} / \mathrm{HA}$ 复合材料的降解速率，实现其在骨科领域的潜在应用。

关 键 词: 牡蚛壳; 羟基磷灰石; 碳酸钙; 降解性能; 骨科

中图分类号: TQ342 文献标识码: A

\section{Preparing Biomedical $\mathrm{CaCO}_{3} / \mathrm{HA}$ Composite with Oyster Shell}

\author{
LIU Ziyang, GENG Zhen, LI Zhaoyang
}

(School of Materials Science and Engineering, Tianjin University, Tianjin 300350, China)

\begin{abstract}
To reduce the cost of HA and improve degradation performance of HA, oyster shells were used as raw materials to prepare $\mathrm{CaCO}_{3}$ / hydroxyapatite (HA) composite materials by a hydrothermal method. Phase analysis and SEM/TEM observation revealed that as-prepared $\mathrm{CaCO}_{3} / \mathrm{HA}$ composites had a morphology of HA nanoparticles growing on the lamellar $\mathrm{CaCO}_{3}$. Three kinds of $\mathrm{CaCO}_{3} / \mathrm{HA}$ composites $(20 \% \mathrm{HA} 、 40 \% \mathrm{HA} 、 60 \% \mathrm{HA})$ with $\mathrm{HA}$ content of $20 \%, 40 \%$ and $60 \%$ were prepared by controlling the proportion of calcium and phosphorus. The actual content of HA in three obtained different composites, determined by ICP, were $17.52 \%, 34.30 \%$ and $43.24 \%$, respectively. With the increase of HA content, the specific surface area and thermal stability of $\mathrm{CaCO}_{3} / \mathrm{HA}$ composite are improved. Samples were immersed in PBS simulated body fluid for $14 \mathrm{~d}$ to evaluate their in vitro degradation ability. The results showed that degradation rates of three kinds of composites with different HA contents $(20 \% \mathrm{HA}$, $40 \% \mathrm{HA}$ and $60 \% \mathrm{HA}$ ) were $15.2 \%, 12.0 \%$ and $10.8 \%$, respectively. The degradation rate decreased with the increase of HA proportion in the composite. All data indicates that the hydrothermal synthesis of $\mathrm{CaCO}_{3} / \mathrm{HA}$ composite can control the conversion degree of $\mathrm{HA}$ and then adjust the degradation rate of $\mathrm{CaCO}_{3} / \mathrm{HA}$ composite by designing the ratio of calcium and phosphorus elements, which have potential application in orthopedics.
\end{abstract}

Key words: oyster shell; hydroxyapatite; calcium carbonate; degradation rate; orthopedic application

收稿日期: 2019-04-04; 收到修改稿日期：2019-04-24

基金项目: 国家自然科学基金(31570970) National Natural Science Foundation of China (31570970)

作者简介：刘子阳(1994-), 男, 硕士研究生. E-mail: 2363664573@qq.com

LIU Ziyang (1994-), male, Master candidate. E-mail: 2363664573@qq.com

通讯作者: 李朝阳，教授. E-mail: zyli@tju.edu.cn

LI Zhaoyang, professor. E-mail: zyli@tju.edu.cn 
羟基磷灰石 $(\mathrm{HA})$ 是钙磷灰石 $\mathrm{Ca}_{5}\left(\mathrm{PO}_{4}\right)_{3}(\mathrm{OH})$ 的 自然矿物化产物, 具有良好的生物相容性、吸附性 能和骨传导性, 目前在生物医学领域有着广泛的应 用 ${ }^{[1-3]}$ 。以钻磷盐作为原料, 传统制备 HA 的方法 ${ }^{[4]}$ 原材料价格较高、产率较低, 在成本与生产效率等 方面都有较大的提升空间。另一方面, HA 虽能在体 内降解成钙、磷离子, 但降解周期较高分子材料更 长, 不能及时地为新生骨提供必需的生长空间。因 此 HA 作为骨修复材料以及药物载体材料的应用受 到了限制 ${ }^{[5]}$ 。

海洋贝类的壳是日常生活中常见的废弃物。以 牡蚛壳为例, 其无机成分以文石(Aragonite)或方解 石(Calcite)型碳酸钲 $\left(\mathrm{CaCO}_{3}\right)$ 为主, 含量达 $95 \%$ 以上, 另有 $\mathrm{Na} 、 \mathrm{Mg} 、 \mathrm{Si} 、 \mathrm{Sr}$ 等多种微量元素, 生蚛壳的降 解产物 $\mathrm{Ca}^{2+} 、 \mathrm{CO}_{3}{ }^{2-}$ 均为人体体液中的常见离子成分, 对机体无明显细胞毒性及遗传毒性, 具有较好的生 物相容性 ${ }^{[6-8]}$ 。 $\mathrm{CaCO}_{3}$ 本身即具有良好的生物活性和 优异的降解性能。朱英杰等 ${ }^{[9]}$ 制备的多孔纳米碳酸 钙空心微球具有良好药物传输性能。但 $\mathrm{CaCO}_{3}$ 初始 强度低、降解速率过快, 在生物医学领域的应用有 限 ${ }^{[10]}$ 。然而, 以牡蚛壳为原材料采用水热法制备的 纳米带状 $\mathrm{HA}^{[11]}$ 、制备的多孔 HA 骨修复材料 ${ }^{[12]}$, 均 显示出以牡蚛壳作为钙源制备 HA 的低成本、低污 染和富含微量元素的优点。同时, 碳酸钻也具有良 好的生物活性 ${ }^{[13]}$, 作为牡蚛壳的主要组成成分, 在 人体内的降解速率远高于纯 HA。因此, 将牡蚛壳与 $\mathrm{HA}$ 复合, 有可能制备出降解速度可调、生物相容性 好、廉价而无污染的骨修复材料, 但目前尚未见相 关报道。

本研究以牡蚛壳为原料, 通过控制不同配比的 钙源与磷源, 探究合成 $\mathrm{CaCO}_{3} / \mathrm{HA}$ 复合材料的理化 性质、降解性能的规律。

\section{1 材料与方法}

\section{1 实验原料准备}

本实验以山东荣成海水养殖的太平洋牡蚛 (Crassostrea gigas)壳为钙源、 $\left(\mathrm{NH}_{4}\right)_{2} \mathrm{HPO}_{4}$ (分析纯, 天津市光复精细化工研究所)为磷源制备 $\mathrm{CaCO}_{3} / \mathrm{HA}$ 复合材料。利用稀释后的浓盐酸(分析纯, 上海市阿 拉丁生化科技有限公司) 清洗牡蚛壳表面, 利用浓 硝酸(分析纯，上海市阿拉丁生化科技有限公司)配 置硝解液, 利用 $\mathrm{NH}_{3} \cdot \mathrm{H}_{2} \mathrm{O}$ (分析纯, 天津市光复精细 化工研究所)调节水热反应溶液的 $\mathrm{pH}$ 。

\section{2 牡蚛壳的预处理}

首先用稀盐酸浸洗牡蚛壳样品, 并用毛刷对牡
蚛壳表面进行清洁, 尽可能去除贝壳上的有机物, 之后再用蒸馏水清洗牡蚛壳表面两次, 得到较清洁 的牡蚛壳样品。 $80{ }^{\circ} \mathrm{C}$ 干燥 $10 \mathrm{~h}$ 后, 放入马弗炉中, $600{ }^{\circ} \mathrm{C}$ 煅烧 $8 \mathrm{~h}$, 升温速率 $10{ }^{\circ} \mathrm{C} / \mathrm{min}$, 使牡蚛壳中 的有机物成分分解, 得到较为纯净的 $\mathrm{CaCO}_{3}$ 钙源。 在炉中冷却至室温后取出, 将干燥的粉末置氧化铝 坩埚中研磨待用。

\section{$1.3 \mathrm{CaCO}_{3} / \mathrm{HA}$ 复合材料的制备}

为了比较不同比例的 $\mathrm{HA}$ 与 $\mathrm{CaCO}_{3}$ 混合产物的 理化性质、核壳结构和降解性能, 本研究做了三组平 行样: $\mathrm{CaCO}_{3}$ 与 $\mathrm{HA}$ 比例为 $8: 2$ 的混合物 $(20 \% \mathrm{HA})$, $\mathrm{CaCO}_{3}$ 与 $\mathrm{HA}$ 比例为 $6: 4$ 的混合物 $(40 \% \mathrm{HA}), \mathrm{CaCO}_{3}$ 与 HA 比例为 $4: 6$ 的混合物 $(60 \% \mathrm{HA})$ 。

$20 \% \mathrm{HA}$ 的制备: 将前述研磨好的粉末 $\left(\mathrm{CaCO}_{3}\right)$ 和 $\left(\mathrm{NH}_{4}\right)_{2} \mathrm{HPO}_{4}$ 以 $50: 6$ 的摩尔比分别溶于 $80 \mathrm{~mL}$ 去 离子水中, 然后用 $\mathrm{NH}_{3} \cdot \mathrm{H}_{2} \mathrm{O}$ 调节混合溶液的 $\mathrm{pH}$ 稳 定在 10 左右，摚拌 $30 \mathrm{~min}$, 将溶液移入反应釜中, 放入恒温干燥箱内在 $150{ }^{\circ} \mathrm{C}$ 的环境下进行水热反 应 $10 \mathrm{~h} 。 40 \% \mathrm{HA}$ 的制备: 研磨好的粉末 $\left(\mathrm{CaCO}_{3}\right)$ 和 $\left(\mathrm{NH}_{4}\right)_{2} \mathrm{HPO}_{4}$ 的摩尔比为 $25: 6$ 。60\%HA 的制备: 粉 末 $\left(\mathrm{CaCO}_{3}\right)$ 与 $\left(\mathrm{NH}_{4}\right)_{2} \mathrm{HPO}_{4}$ 的摩尔比为 $50: 18$ 。水热 反应结束后, 通过离心得到白色沉淀物, 并用去离 子水和无水乙醇反复清洗两次, 最后在 $70{ }^{\circ} \mathrm{C}$ 干燥 $24 \mathrm{~h}$ 得到 $\mathrm{CaCO}_{3} / \mathrm{HA}$ 复合样品。

采用 X-ray Diffraction(XRD)分析牡蚄壳煅烧 产物与水热产物的物相。采用 Brunauer-EmmettTeller (BET)模型分析测定产物的比表面积, 样品预 热至 $100{ }^{\circ} \mathrm{C}$ 后，置入氮气气氛中 $24 \mathrm{~h}$, 利用孔径分 布测试仪进行检测。用扫描电子显微镜(SEM)、透 射电子显微镜(TEM)观察和 $X$ 射线能谱仪(EDS)分 析试样的形貌及元素含量。用热重分析仪(TGA)分 析(样品 $30 \mathrm{mg}$, 升温速率 $10{ }^{\circ} \mathrm{C} / \mathrm{min}$ ) 样品的热稳 定性。利用 Inductively coupled plasma (ICP)测试样 品中的钙、磷元素含量。

\section{$1.4 \mathrm{CaCO}_{3} / \mathrm{HA}$ 复合材料的体外降解实验}

将三组样品分别取三份, 每组 $5 \mathrm{mg}$, 并添加煅 烧清洗后的牡蚛壳作为对照, 准备进行体外模拟体 液降解实验。将各组试样分别置于 $15 \mathrm{~mL}$ 离心管中, 每个离心管中加入 $10 \mathrm{~mL}$ 的磷酸盐缓冲生理盐水 (PBS)，放在摇床上捊育，温度恒定在 $36.8{ }^{\circ} \mathrm{C}$ 。于 7 、 $14 、 21 \mathrm{~d}$ 分别取出。取出后通过离心分离样品与 PBS 培养液, 将剩余样品置于 $60{ }^{\circ} \mathrm{C}$ 的电热干燥箱 内干燥至恒重, 然后保存待用。PBS 培养液同样保 存待用。

利用 ICP 测试样品 PBS 浸泡液中的钙元素含 
量, 通过对比初始样品以及降解不同时间后所得 浸泡液的䥻离子浓度, 可以得到不同转化程度样品 的降解速率。取一定量各组剩余的降解试样, 分别 置于 $15 \mathrm{~mL}$ 离心管中, 每个离心管中加入 $10 \mathrm{~mL}$ 的 稀硝酸, 搅拌溶解之后利用 ICP 测试其钙元素含量, 通过计算可以得到样品中 $\mathrm{CaCO}_{3}$ 与 $\mathrm{HA}$ 的降解比 例。最后取少量降解后样品, 干燥喷金之后用 SEM 观察形貌。

\section{2 结果与讨论}

\section{1 牡蚛壳煅烧产物物相}

牡蚛壳的㷽烧产物首先利用 ICP 测试其中的重 金属含量, 所得结果如表 1 所示, 符合国家标准 GB 23101 的要求。

牡蚛壳经过清洗煅烧等处理后所得粉末的物相

表 1 牡蚛壳煅烧产物的重金属含量

Table 1 Heavy metal content of the calcined products of oyster shells

\begin{tabular}{ccc}
\hline Element & Calcined product $/\left(\mathrm{mg} \cdot \mathrm{kg}^{-1}\right)$ & $\mathrm{Limit} /\left(\mathrm{mg} \cdot \mathrm{kg}^{-1}\right)$ \\
\hline $\mathrm{Pb}$ & $<10$ & 30 \\
$\mathrm{Cd}$ & $<1.5$ & 5 \\
$\mathrm{Hg}$ & $<1$ & 5 \\
$\mathrm{As}$ & $<1$ & 3 \\
\hline
\end{tabular}
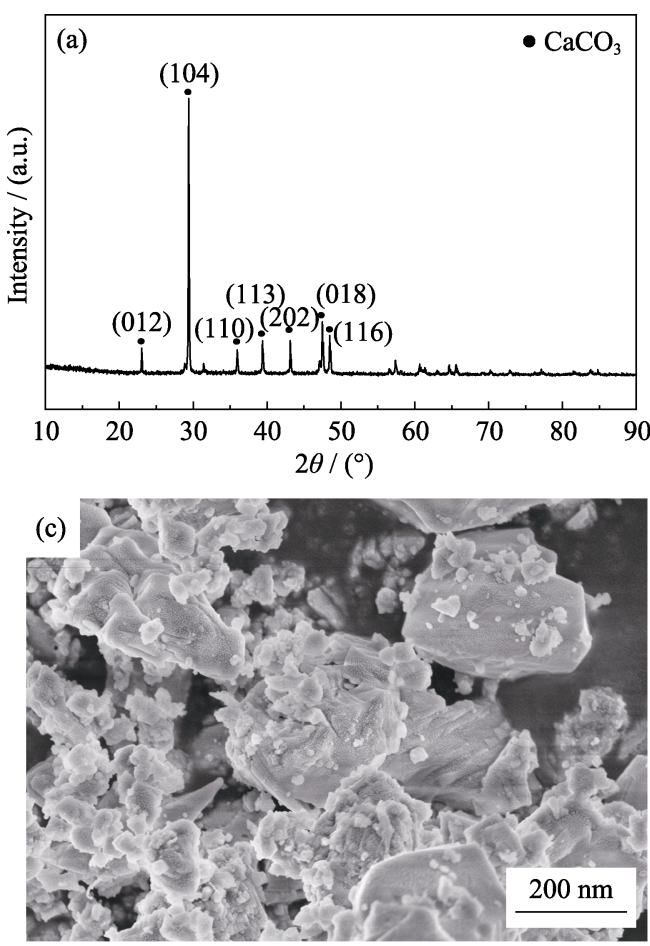

表征结果如图 1 所示。图 1(a)是样品的 XRD 图谱, 可见牡蚛壳㷽烧产物的主要物相是 $\mathrm{CaCO}_{3}(\mathrm{JCPDS}$ 47-1743), 产物对应特征峰与方解石型碳酸钙相符。 从 SEM 结果可以看出, 牡蚛壳炦烧水洗之后的产 物呈片层状结构(图 1(c)), 多为大小在 $200 \mathrm{~nm}$ 至 $500 \mathrm{~nm}$ 左右的片状结构堆积而成。查阅相关文献可 知 ${ }^{[6-8]}$, 方解石型碳酸钙显微照片即为片层状的堆 积结构, 与实验结果相符。从 EDS 分析结果(图 1(b)) 可以看出, 殷烧产物主要组成元素为碳、氧、钙元 素，与预期的碳酸钻成分相符; 同时含有微量的 $\mathrm{Mg}$ 元素, 这可能是贝壳中含有少量 $\mathrm{Mg}(\mathrm{OH})_{2}$ 导致 的结果 ${ }^{[14]}$ 。TEM 照片显示的结果与 SEM 照片一致, 煅烧产物由 200 500 $\mathrm{nm}$ 的片状结构组成, 片层之 间相互堆积程度较为密集, 选区电子衍射(SAED)图 像显示样品是一种典型的单晶结构, 具有良好的结 晶度, 与 $\mathrm{XRD}$ 结果一致。

\section{$2.2 \mathrm{CaCO}_{3} / \mathrm{HA}$ 复合材料的物相表征}

图 2 为复合产物的 XRD 图谱, 三者分别对应 $\mathrm{HA}$ 转化程度为 $20 \% 、 40 \%$ 与 $60 \%$ 的产物。从图谱 中可以看出, 三者的物相均对应了 HA (JCPDS 03-0747) 与方解石型 $\mathrm{CaCO}_{3}(\mathrm{JCPDS}$ 47-1743)两套 不同的衍射峰。而随着 HA 转化程度的提高, 图谱 中 $\mathrm{CaCO}_{3}$ 所对应的特征峰峰强度有下降，同时, $\mathrm{HA}$ 对应的特征峰强度提高。样品的峰型明显, 结晶度 良好。
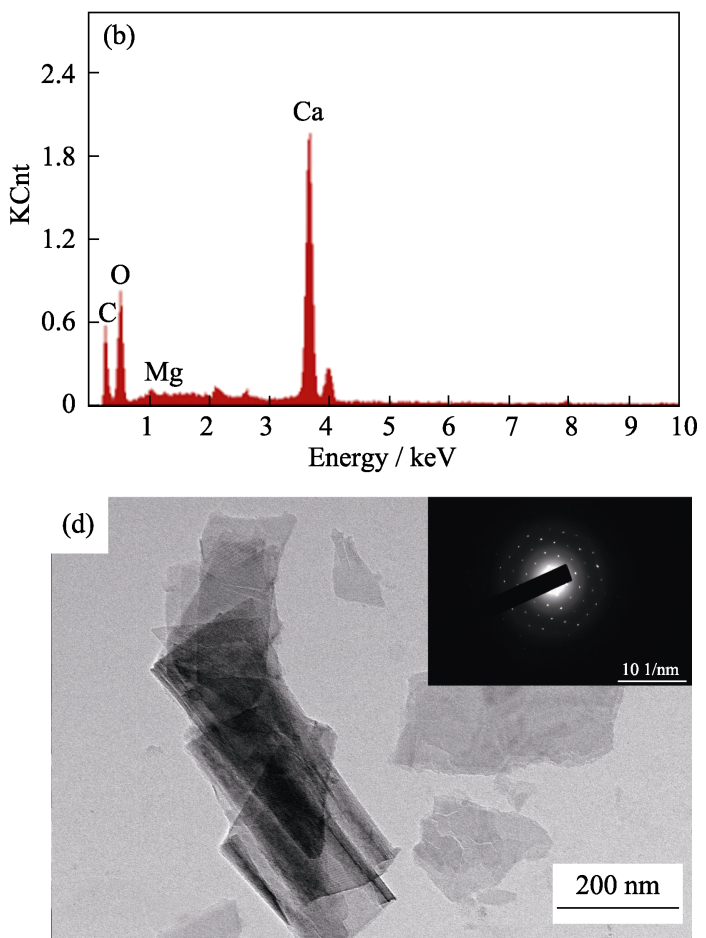

图 1 牡蚛壳经㷽烧水洗后所得粉末的(a)XRD 图谱、(b)EDS 分析结果、(c)SEM 照片和(d)TEM 照片及 SAED 图谱

Fig .1 (a) XRD pattern, (b) EDS analysis, (c) SEM micrograph and (d) TEM image and SAED pattern of the powders prepared by heating and washing the oyster shell 


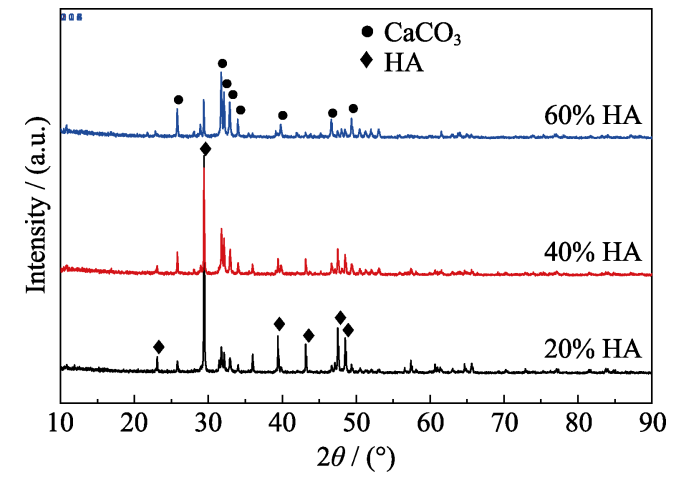

图 2 水热产物的 XRD 图谱

Fig. 2 XRD patterns of of the synthesized samples

利用 ICP 表征样品中 $\mathrm{Ca}$ 元素与 $\mathrm{P}$ 元素的含量。 将制得的三种样品各取 $5 \mathrm{mg}$, 溶解于 $100 \mathrm{~mL} \mathrm{HNO}_{3}$ 中制备了对应溶液, 之后利用 ICP 测试溶液中 $\mathrm{Ca}$ 元素与 $\mathrm{P}$ 元素的浓度, 结果如表 2 所示。根据之前 的 XRD 结果, 可认为 $\mathrm{HA}$ 与 $\mathrm{CaCO}_{3}$ 是样品的主要 组成成分。因此通过计算就能得到所制备的三种样 品中 $\mathrm{HA}$ 的转化程度, 分别为 $(17.52 \pm 0.57) \%$ 、 $(34.30 \pm 1.02) \%$ 、(43.24 \pm 0.33$) \%$ 。

\section{表 2 样品中 Ca、P 离子含量及 HA 转化程度 \\ Table 2 Ca and $P$ concentrations and $H A$ transition degree of samples}

\begin{tabular}{cccc}
\hline & $20 \% \mathrm{HA}$ & $40 \% \mathrm{HA}$ & $60 \% \mathrm{HA}$ \\
\hline $\begin{array}{c}\text { Concentration } \\
\text { of } \mathrm{Ca} /\left(\mathrm{mg} \cdot \mathrm{L}^{-1}\right)\end{array}$ & $(19.91 \pm 0.72)$ & $(20.00 \pm 1.08)$ & $(20.02 \pm 0.52)$ \\
$\begin{array}{c}\mathrm{Concentration} \\
\text { of } \mathrm{P} /\left(\mathrm{mg} \cdot \mathrm{L}^{-1}\right)\end{array}$ & $(1.62 \pm 0.17)$ & $(3.19 \pm 0.22)$ & $(4.02 \pm 0.19)$ \\
$\begin{array}{c}\text { Degree of HA } \\
\text { transition } / \%\end{array}$ & $(17.5 \pm 0.57)$ & $(34.3 \pm 1.02)$ & $(43.2 \pm 0.33)$ \\
\hline
\end{tabular}

从以上数据可以看出, 当样品磷源投料比例在 $20 \%$ 与 $40 \%$ 时, 样品的转化程度与预期结果比较 接近, 说明样品的转化过程比较成功; 而当磷源投 料比例提高到 $60 \%$ 时, 样品的转化程度稍差, 这 可能是因为采用的 $\mathrm{CaCO}_{3}$ 样品显微结构是片层状
的堆积形貌 ${ }^{[15]}$, 比表面积相对较小, 导致反应的 接触面积较小，因此反应得不够完全。整体上来看 样品的取代比例控制在 $40 \%$ 以下时，转化反应率 较高。

图 3 为合成 $\mathrm{CaCO}_{3} / \mathrm{HA}$ 复合产物的 SEM 照片。 图 3(a)为 $20 \% \mathrm{HA}$ 样品的 SEM 照片, 可以观察到样 品呈现为片层状的堆积结构, 与 $\mathrm{CaCO}_{3}$ 的显微结构 十分相似, 综合前文 XRD 与 ICP 的测试结果, 可以 证明样品保留了碳酸钙的基本结构, 而在此基础上, 有少量的 $\mathrm{HA}$ 纳米颗粒在片层状 $\mathrm{CaCO}_{3}$ 的表面生长, 由于 HA 的转化程度较低, 故 HA 纳米颗粒的含量 较少, 形貌不明显。

图 3(b)为 40\%HA 样品的 SEM 照片, 其显微形 貌同样表现为片层状的堆积结构上生长出颗粒状的 HA，随着 HA 转化程度的提高, HA 颗粒的密集程度 有明显的提高; 而对于 $60 \% \mathrm{HA}$ (图 3(c))样品, 样品 片层状结构表面的 HA 更加密集。根据以上实验结 果, 制得的三种样品均为在 $\mathrm{CaCO}_{3}$ 结构的表面有 $\mathrm{HA}$ 颗粒生成, 而随着 HA 转化程度的提升, HA 颗 粒的密度明显提高，结果符合实验预期。

图 4 为所得样品的 TEM 与高倍 TEM 照片。从 图 4(a)中可以看出 $20 \% \mathrm{HA}$ 的形貌为片层状结构上 有少量的纳米颗粒 HA 生成，片层尺寸为(100 $150 \mathrm{~nm}) \times 400 \mathrm{~nm}$, 结晶度良好。从图 4(c)中可以看 出 $40 \% \mathrm{HA}$ 的形貌为片层状结构上有大量的纳米颗 粒 HA 生成，团聚性较强，纳米颗粒大小在 10 20 nm 之间，结晶度良好，由于 $\mathrm{HA}$ 纳米颗粒的团聚， HRTEM 显示的结构为多晶结构。从图 4(e)中可以看 出 $60 \% \mathrm{HA}$ 的 TEM 形貌为片层状结构上大量生长出 $\mathrm{HA}$ ，其形貌发生了从纳米颗粒状向条棒状的转变, 团聚现象明显，晶粒尺寸为 $(20 \sim 50 \mathrm{~nm}) \times 5 \mathrm{~nm}$ 。这些 结果与 XRD 和 SEM 分析结果相一致。

图 5 为所得样品的 BET 比表面积测试结果, $20 \% \mathrm{HA} 、 40 \% \mathrm{HA}$ 和 $60 \% \mathrm{HA}$ 的比表面积分别为 $(18.8 \pm 1.8) 、(30.2 \pm 2.4)$ 和 $(36.2 \pm 2.6) \mathrm{m}^{2} / \mathrm{g}$ 。由于
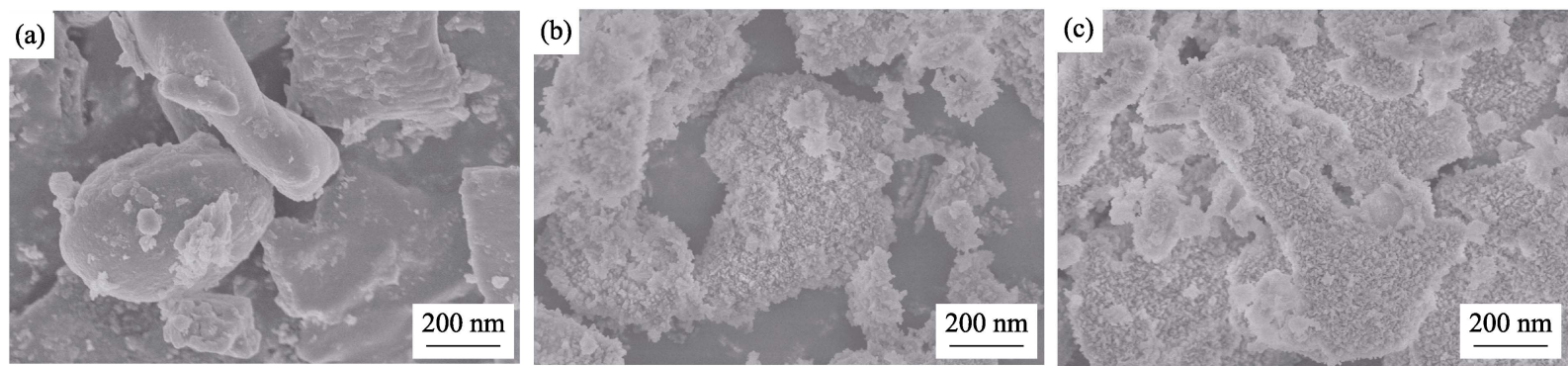

图 3 水热产物的 SEM 形貌照片

Fig. 3 SEM micrographs of the hydrothermally synthesized samples (a) $20 \% \mathrm{HA}$; (b) $40 \% \mathrm{HA}$; (c) $60 \% \mathrm{HA}$ 

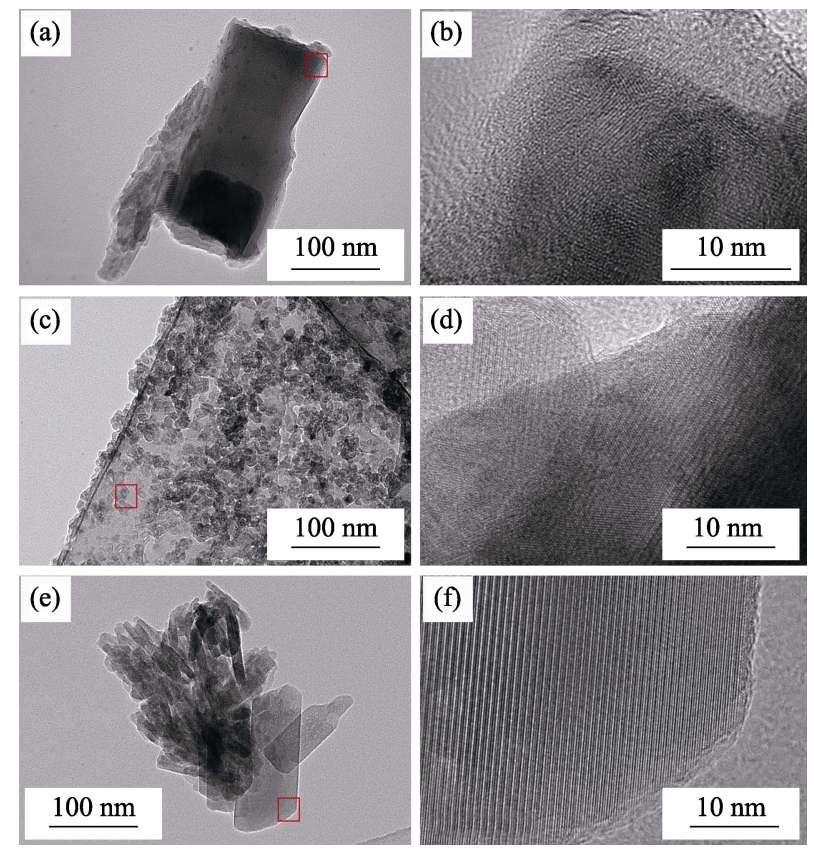

图 4 水热产物的 TEM 和 HRTEM 照片

Fig. 4 TEM and HRTEM images of the hydrothermally synthesized samples

(a, b) $20 \% \mathrm{HA}$; (c, d) $40 \% \mathrm{HA}$; (e, f) $60 \% \mathrm{HA}$

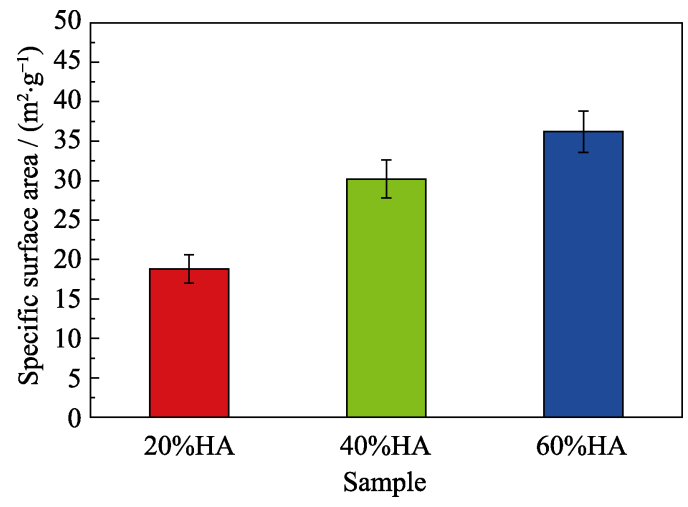

图 5 水热产物 BET 测试结果

Fig. 5 Specific surface area of the prepared samples

$\mathrm{CaCO}_{3}$ 呈片层状结构的比表面积要显著低于颗粒状 $\mathrm{HA}^{[16]}$, 因此比表面积的数值越大, 说明该材料微 观结构中的 HA 纳米颗粒的含量越高。从 BET 的测 试结果来看, 随着 HA 转化程度的提高, 样品的比 表面积显著提高, 这与图 3 样品 SEM 形貌照片的实
验结果相吻合。

热重分析结果如图 6 所示。低于 $200{ }^{\circ} \mathrm{C}$ 和 $400{ }^{\circ} \mathrm{C}$ 的失重主要是由于样品中吸附水和结晶水的 蒸发 ${ }^{[17]}$ 。在低于 $200{ }^{\circ} \mathrm{C}$ 范围的时候三种样品的失重 率没有明显的变化, 说明三者的吸附水含量相似。

在 $400{ }^{\circ} \mathrm{C}$ 以上的温度条件下 $\mathrm{HA}$ 会发生磷酸氢根的 分解。650 ${ }^{\circ} \mathrm{C}$ 以上的失重速率的明显变化可能是由 于 $\mathrm{CaCO}_{3}$ 的分解: $\mathrm{CaCO}_{3} \rightarrow \mathrm{CaO}+\mathrm{CO}_{2}$, 可以看出, $\mathrm{HA}$ 转化程度较低的样品在这个范围内表现出了更 快的分解速度以及更大的失重。 $\mathrm{HA}$ 在 $660 \sim 800{ }^{\circ} \mathrm{C}$ 之间会产生新的分解: $2 \mathrm{Ca}_{5}\left(\mathrm{PO}_{4}\right)_{3}(\mathrm{OH}) \rightarrow 3 \mathrm{Ca}_{3}\left(\mathrm{PO}_{4}\right)_{2}+$ $\mathrm{CaO}+\mathrm{H}_{2} \mathrm{O}^{[18]}$ 。所有试样的热稳定性较高, 总失重均 小于 $12 \%$ 。

\section{$2.3 \mathrm{CaCO}_{3} / \mathrm{HA}$ 复合材料降解性}

表 3 是样品经降解实验后离心所得的 PBS 上清 液中钙元素的含量。传统的 HA 在人体内的降解速 率为 $14 \mathrm{~d}$ 降解 2.4\% 3.6\% ${ }^{[19-21]}$, 而通过计算得到的 三种样品在 PBS 模拟体液中的降解速率见表 3 。可 以发现制得的 $\mathrm{CaCO}_{3} / \mathrm{HA}$ 复合材料在降解性能上有 了明显的改善, $20 \% \mathrm{HA} 、 40 \% \mathrm{HA}$ 和 $60 \% \mathrm{HA}$ 三种不 同 HA 含量的复合材料在 PBS 模拟体液中 $14 \mathrm{~d}$ 的 降解速率分别为 $15.2 \% 、 12.0 \%$ 和 $10.8 \%$, 降解速率随 $\mathrm{HA}$ 在材料中所占的比例增加而降低。

图 7 为降解实验后回收样品的 SEM 照片。如 图 7(a)所示, $20 \% \mathrm{HA}$ 样品在 PBS 模拟体液中浸泡 $7 \mathrm{~d}$

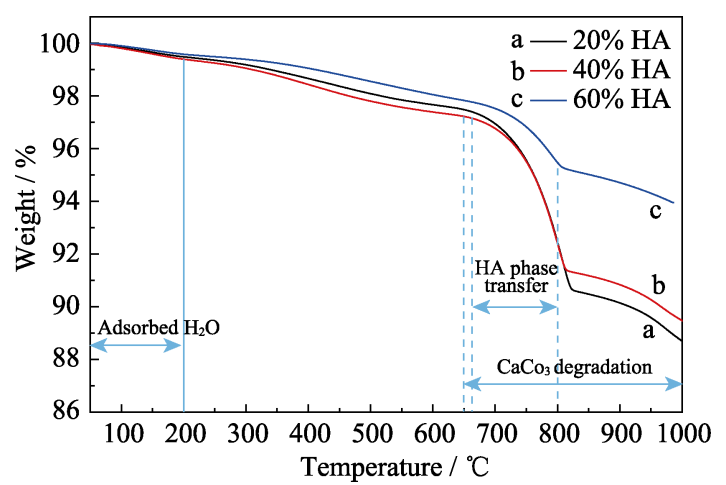

图 6 样品热失重的曲线图

Fig. 6 TG curves of the prepared samples

表 3 样品降解释放 $\mathrm{Ca}^{2+}$ 浓度及降解率

Table $3 \mathrm{Ca}^{2+}$ concentration and degradation rate of samples

\begin{tabular}{|c|c|c|c|c|c|c|c|c|}
\hline & \multicolumn{2}{|c|}{$20 \% \mathrm{HA}$} & \multicolumn{2}{|c|}{$40 \% \mathrm{HA}$} & \multicolumn{2}{|c|}{$60 \% \mathrm{HA}$} & \multicolumn{2}{|c|}{$\mathrm{CaCO}_{3}$} \\
\hline $\begin{array}{l}\text { Degradation } \\
\text { time/d }\end{array}$ & $\begin{array}{l}\text { Concentration } \\
\text { of } \\
\mathrm{Ca}^{2+} /\left(\mathrm{mg} \cdot \mathrm{L}^{-1}\right)\end{array}$ & $\begin{array}{l}\text { Degradation } \\
\text { rate } / \%\end{array}$ & $\begin{array}{l}\text { Concentration of } \\
\mathrm{Ca}^{2+} /\left(\mathrm{mg} \cdot \mathrm{L}^{-1}\right)\end{array}$ & $\begin{array}{l}\text { f Degradation } \\
\text { rate } / \%\end{array}$ & $\begin{array}{l}\text { Concentration of } \\
\mathrm{Ca}^{2+} /\left(\mathrm{mg} \cdot \mathrm{L}^{-1}\right)\end{array}$ & $\begin{array}{l}\text { Degradation } \\
\text { rate } / \%\end{array}$ & $\begin{array}{l}\text { Concentration of } \\
\mathrm{Ca}^{2+} /\left(\mathrm{mg}^{-1} \mathrm{~L}^{-1}\right)\end{array}$ & $\begin{array}{l}\text { Degradation } \\
\text { rate } / \%\end{array}$ \\
\hline 7 & $(16.13 \pm 0.40)$ & 8.1 & $(13.39 \pm 0.23)$ & 6.7 & $(11.61 \pm 0.92)$ & 5.8 & $(18.93 \pm 0.17)$ & 9.8 \\
\hline 14 & $(30.26 \pm 1.12)$ & 15.2 & $(24.02 \pm 0.83)$ & 12.0 & $(21.62 \pm 1.36)$ & 10.8 & $(42.70 \pm 0.19)$ & 22.1 \\
\hline 21 & $(43.20 \pm 1.47)$ & 21.7 & $(33.40 \pm 1.38)$ & 16.2 & $(28.11 \pm 1.07)$ & 14.3 & $(56.41 \pm 0.32)$ & 29.2 \\
\hline
\end{tabular}



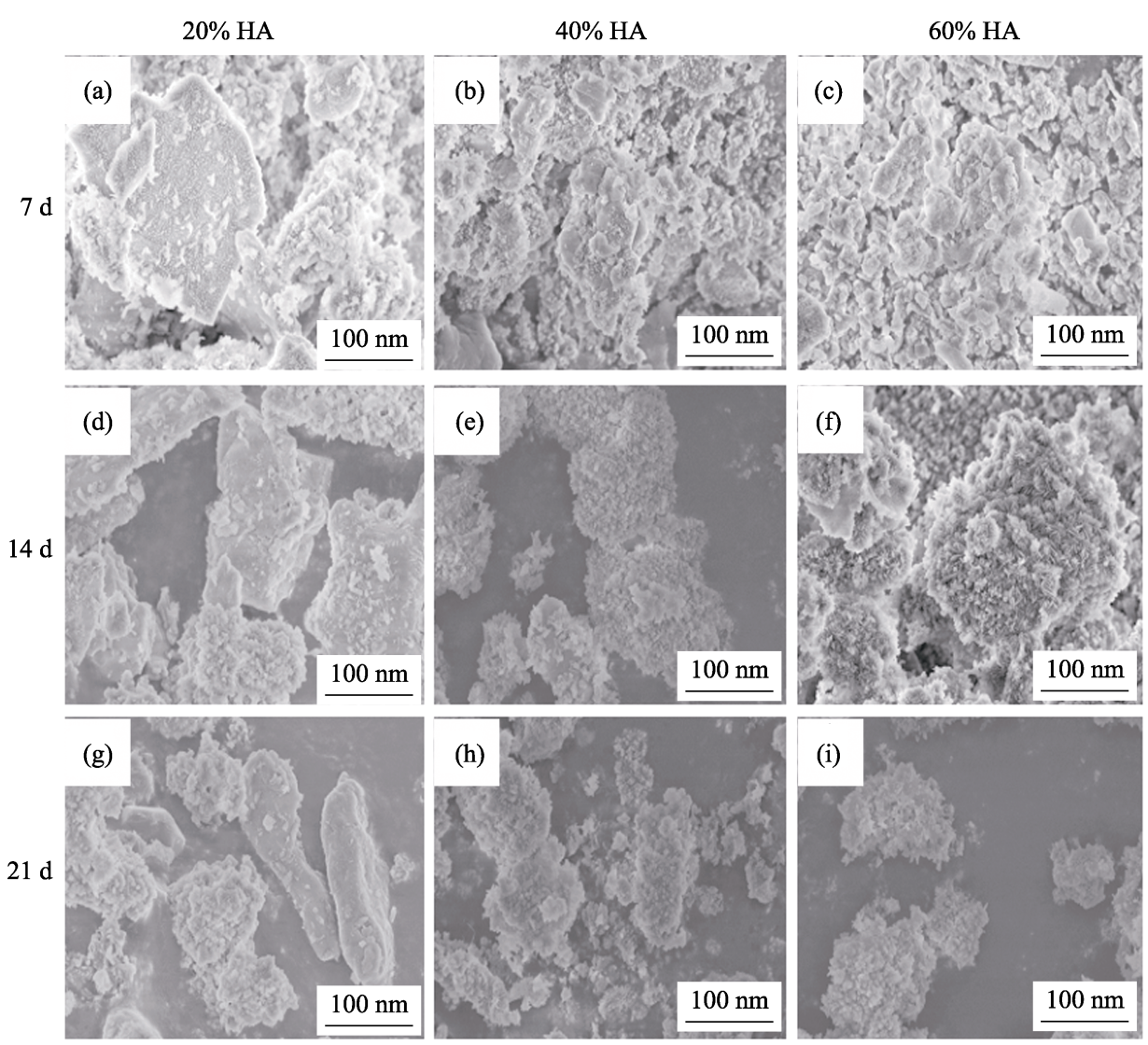

图 7 样品降解试验后的 SEM 照片

Fig. 7 SEM micrographs of the samples after degradation

之后保留了明显的片层状堆积结构, 片层表面有少 量的 HA 颗粒, 同时可以看到片层表面有一定量降 解后的 $\mathrm{CaCO}_{3}$ 碎片残留, 片层状结构的大小为 200 $400 \mathrm{~nm}$ 。20\%HA 样品在 PBS 模拟体液中浸泡 $14 \mathrm{~d}$ 之后，片层状堆积结构依然明显，片层表面的 $\mathrm{CaCO}_{3}$ 碎片增多, 有少量的 $\mathrm{HA}$ 颗粒残留, 片层状 $\mathrm{CaCO}_{3}$ 的堆积密度相比浸泡 $7 \mathrm{~d}$ 的样品有所下降, 尺寸上 也有一定程度的减小, 如图 7(d) 所示。 $20 \% \mathrm{HA}$ 样品 在 PBS 模拟体液中浸泡 $21 \mathrm{~d}$ 之后, 仍然保留了 $\mathrm{CaCO}_{3}$ 的片层状堆积结构, 但密集程度相比 7、14 d 有明显 下降，尺寸上也减小到 100 200 nm，同时片层表面 可以明显地观察到 $\mathrm{CaCO}_{3}$ 碎片, 如图 7(d)所示。

$40 \% \mathrm{HA}$ 与 $60 \% \mathrm{HA}$ 的样品随着在 PBS 溶液中浸 泡时间的延长, 片层状 $\mathrm{CaCO}_{3}$ 的堆积密度有明显的 下降, 尺寸上也有一定程度的减小, 同时片层状 $\mathrm{CaCO}_{3}$ 表面的 $\mathrm{HA}$ 颗粒的密度与尺寸也有明显的下 降。从图 7(f)中可以看到片层状 $\mathrm{CaCO}_{3}$ 表面的 $\mathrm{HA}$ 形 貌由原来的颗粒状向丝状转变, 降解行为明显。

\section{3 结论}

本研究采用牡蚛壳为钙源, 采用水热反应的方 法并通过控制磷源的投入比例制备出 $20 \% \mathrm{HA}$ 、
$40 \% \mathrm{HA}$ 和 $60 \% \mathrm{HA}$ 三种 $\mathrm{CaCO}_{3} / \mathrm{HA}$ 复合样品, 对产 物进行物相表征, 并通过体外降解实验探索样品降 解性能的提升, 结论如下:

1) 制备出的 $20 \% \mathrm{HA} 、 40 \% \mathrm{HA}$ 和 $60 \% \mathrm{HA}$ 三种 样品为 $\mathrm{CaCO}_{3}$ 与 $\mathrm{HA}$ 的复合产物, 具有良好的结晶 度, 显微形貌为片层状的 $\mathrm{CaCO}_{3}$ 结构上有纳米颗粒 状的 HA 生长, 随着转化程度的提高, HA 的密度有 显著提升;

2) 与传统的 $\mathrm{HA}$ 相比, $\mathrm{CaCO}_{3} / \mathrm{HA}$ 复合材料的比 表面积和热稳定性均稍有下降, 随着转化程度的 提高, 复合产物的比表面积增加, 热稳定性也有所 提高;

3) 相比于传统的 $\mathrm{HA}, \mathrm{CaCO}_{3} / \mathrm{HA}$ 复合材料的降 解速率大幅提高, $20 \% \mathrm{HA} 、 40 \% \mathrm{HA}$ 和 $60 \% \mathrm{HA}$ 在 $\mathrm{PBS}$ 模拟体液中 $14 \mathrm{~d}$ 的降解率分别为 $15.2 \% 、 12.0 \%$ 和 $10.8 \%$ 。

\section{参考文献:}

[1] MEHDI S S, KHORASANI M T, EHSAN D K, et al. Synthesis methods for nanosized hydroxyapatite with diverse structures. Acta Biomater., 2013; 9(8): 7591-7621.

[2] BASHA R Y, SAMPATH K T S, DOBLE M. Design of biocomposite materials for bone tissue regeneration. Mater. Sci. Eng. C, 
2015, 57: 452-463.

[3] FACCA S, LAHIRI D, FIORETTI F, et al. In vivo osseointegration of nanodesigned composite coatings on titanium implants. ACS Nano, 2011, 5(6): 4790-4799.

[4] RHAITI H, LAGHZIZIL A, SAOIABI A, et al. Surface properties of porous hydroxyapatite derived from natural phosphate. Mater. Chem. Phys., 2012, 136(2): 1022-1026.

[5] ZHANG C Y, ZHANG S Y, LIU X P, et al. Degradation behavior of $\mathrm{MgF}_{2} / \mathrm{HA}$ composite coating on magnesium alloy in vitro. The Chinese Journal of Nonferrous Metals, 2018, 28(4): 766-773.

[6] LEMOS A F, ROCHA J H G, FERREIRA J M F, et al. Hydroxyapatite nano-powders produced hydrothermally from nacreous material. Journal of the European Ceramic Society. 2006, 26(16): 3639-3646.

[7] VECCCHIO K S, ZHANG X, MASSIE J B, et al. Conversion of bulk seashells to biocompatible hydroxyapatite for bone implants. Acta Biomaterials, 2007, 3(6): 910-918.

[8] LANDi E, CELOTTI G, LOGROSCINO G, et al. Carbonated hydroxyapatite as bone substitute. Journal of the European Ceramic Society, 2003, 23(15): 2931-2937.

[9] LI L, ZHU Y J, CAO S W, et al. Preparation and drug release properties of nanostructured $\mathrm{CaCO}_{3}$ porous hollow microspheres. Journal of Inorganic Materials, 2009, 24(1): 166-170.

[10] GUAN J K, XU Z Y, CHEN B, et al. Preparation and properties of nanometer calcium carbonate/poly (L-lactide) composite materials. Journal of Clinical Rehabilitative Tissue Engineering Research, 2008, 12(10): 1889-1891.

[11] ZHAO H, CHEN Y, LI Z M, et al. Preparation of nanoribbon hydroxyapatite from discarded shells. Gansu Science and Technology, 2016, 32(17): 50-52.

[12] XUE Q H, XING Y Z, ZHANG Q Q. Preparation technology of oyster hydroxyapatite porous material for bone repair. Int. J. Bio- med. Eng., 2018, 41(4): 291-295.

[13] YANG S L, GAO Q, WANG H T. Theoretical prediction of ring strain energies of several carbon-monocyclic and carbon-dicyclic compounds.Journal of Natural Science of Heilongjiang University, 2010, 27(4): 495-499.

[14] RUJITANAPANICH S, KUMPAPAN P, WANJANOI P, et al. Synthesis of hydroxyapatite from oyster shell via precipitation method. Energy Proc., 2014, (56): 112-117.

[15] AKRAM M, AHMED R, SHAKIR I, et al. Extracting hydroxyapatite and its precursors from natural resources. J. Mater. Sci. 2014; 49(4): 1461-1475.

[16] NAYAR S, GUHA A. Waste utilization for the controlled synthesis of nanosized hydroxyapatite. Mater. Sci. Eng., 2009, 29(4): $1326-1329$.

[17] LANDI E, SPRIO S, SANDRI M, et al. Development of Sr and $\mathrm{CO}_{3}$ co-substituted hydroxyapatites for biomedical applications. Acta Biomaterialia, 2008, 4(3): 656-663.

[18] MEEJOO S, MANEEPRAKORN W, WINOTAI P. Phase and thermal stability of nanocrystalline hydroxyapatite prepared via microwave heating. Thermochimica Acta, 2006, 447(1): 115-120.

[19] HU GF, XIAO LW, FU H, et al. Study on injectable and degradable cement of calcium sulphate and calcium phosphate for bone repair. Journal of Materials Science: Materials in Medicine, 2010, 21(2): 627-634.

[20] QI X, YE J, WANG Y. Improved injectability and in vitro degradation of a calcium phosphate cement containing poly (lactide-co- glycolide) microspheres. Acta Biomaterialia, 2008, 4(6): 1837- 1845.

[21] SRINATH PALAKURTHY, VENU GOPAL REDDY K, RAJ KUMAR SAMUDRALA, et al. In vitro bioactivity and degradation behaviour of $\beta$-wollastonite derived from natural waste. Materials Science \& Engineering, 2019, 98: 109-117. 